\title{
Article \\ Phylogenetic and Molecular Analyses of More Prevalent HCV1b Subtype in the Calabria Region, Southern Italy
}

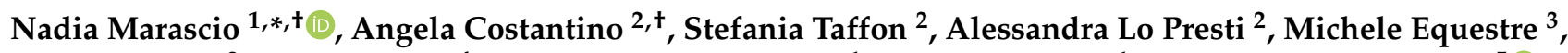 \\ Roberto Bruni ${ }^{2}$, Giulio Pisani ${ }^{4}$, Giorgio Settimo Barreca ${ }^{1}$, Angela Quirino ${ }^{1}$, Enrico Maria Trecarichi ${ }^{5}{ }^{\circ}$, \\ Chiara Costa ${ }^{5}$, Maria Mazzitelli ${ }^{5}{ }^{(0}$, Francesca Serapide ${ }^{5}$, Giovanni Matera ${ }^{1}$, Carlo Torti ${ }^{5}{ }^{(}$, Maria Carla Liberto ${ }^{1}$ \\ and Anna Rita Ciccaglione ${ }^{2}$
}

check for

updates

Citation: Marascio, N.; Costantino, A.; Taffon, S.; Lo Presti, A.; Equestre, M.; Bruni, R.; Pisani, G.; Barreca, G.S.; Quirino, A.; Trecarichi, E.M.; et al. Phylogenetic and Molecular Analyses of More Prevalent HCV1b Subtype in the Calabria Region, Southern Italy. J. Clin. Med. 2021, 10, 1655. https:// doi.org/10.3390/jcm10081655

Academic Editor: Yasuhito Tanaka

Received: 2 March 2021

Accepted: 8 April 2021

Published: 13 April 2021

Publisher's Note: MDPI stays neutral with regard to jurisdictional claims in published maps and institutional affiliations.

Copyright: (c) 2021 by the authors. Licensee MDPI, Basel, Switzerland. This article is an open access article distributed under the terms and conditions of the Creative Commons Attribution (CC BY) license (https:// creativecommons.org/licenses/by/ $4.0 /)$.
1 Department of Health Sciences, Institute of Microbiology, "Magna Grecia" University, 88100 Catanzaro, Italy; gbarreca@unicz.it (G.S.B.); quirino@unicz.it (A.Q.); gm4106@gmail.com (G.M.); mliberto@unicz.it (M.C.L.)

2 Department of Infectious Diseases, Istituto Superiore di Sanità, 00161 Rome, Italy; angela.costantino@iss.it (A.C.); stefania.taffon@iss.it (S.T.); alessandra.lopresti@iss.it (A.L.P.); roberto.bruni@iss.it (R.B.); annarita.ciccaglione@iss.it (A.R.C.)

3 Department of Cell Biology and Neuroscience, Istituto Superiore di Sanità, 00161 Rome, Italy; michele.equestre@iss.it

4 National Center for Immunobiologicals Research and Evaluation, Istituto Superiore di Sanità, 00161 Rome, Italy; giulio.pisani@iss.it

5 Department of Medical and Surgical Sciences, Unit of Infectious and Tropical Diseases, “Magna Graecia" University, 88100 Catanzaro, Italy; em.trecarichi@unicz.it (E.M.T.); c.costa@materdominiaou.it (C.C.); m.mazzitelli88@gmail.com (M.M.); francescaserapide@gmail.com (F.S.); torti@unicz.it (C.T.)

* Correspondence: nmarascio@unicz.it; Tel.: +39-0961-3697-742; Fax: +39-0961-3697-760

+ Contributed equally.

Abstract: Hepatitis C virus subtype $1 \mathrm{~b}$ (HCV1b) is still the most prevalent subtype worldwide, with massive expansion due to poor health care standards, such as blood transfusion and iatrogenic procedures. Despite safe and effective new direct antiviral agents (DAA), treatment success can depend on resistance-associated substitutions (RASs) carried in target genomic regions. Herein we investigated transmission clusters and RASs among isolates from HCV1b positive subjects in the Calabria Region. Forty-one NS5B and twenty-two NS5A sequences were obtained by Sanger sequencing. Phylogenetic analysis was performed using the maximum likelihood method and resistance substitutions were analyzed with the Geno2pheno tool. Phylogenetic analysis showed sixteen statistically supported clusters, with twelve containing Italian sequences mixed with foreign $\mathrm{HCV} 1 \mathrm{~b}$ isolates and four monophyletic clusters including only sequences from Calabria. Interestingly, HCV1b spread has been maintained by sporadic infections in geographically limited areas and by dental treatment or surgical intervention in the metropolitan area. The L159F NS5B RAS was found in 15 isolates and in particular 8/15 also showed the C316N substitution. The Y93H and L31M NS5A RASs were detected in three and one isolates, respectively. The A92T NS5A RAS was found in one isolate. Overall, frequencies of detected NS5B and NS5A RASs were $36.6 \%$ and $22.7 \%$, respectively. For the eradication of infection, improved screening policies should be considered and the prevalence of natural RASs carried on viral strains.

Keywords: hepatitis C virus (HCV); phylogeny; resistance-associated substitution (RAS)

\section{Introduction}

Hepatitis C (HCV) infection remains a major public health problem, even if in the last few years HCV therapy has been improved by the availability of direct-acting antiviral (DAA) agents [1]. Phylogenetic analyses have identified eight HCV major genotypes, further subdivided into 67 subtypes [2]. HCV1b is widespread all over the world, HCV2 showed higher prevalence in Russia and in Italy. In Europe, the most common HCV2 
subtypes are HCV2a/2c. HCV1a and HCV3a predominate in Europe and North America, while HCV4 is endemic in the Middle East, Central Africa and Mediterranean countries. HCV5 is endemic in South Africa, HCV6 in South East Asia and HCV7 was found in the Democratic Republic of Congo. Recently, HCV8 was found in Indian patients living in Canada [2-4]. Magiorkinis and colleagues reported a massive expansion of HCV1b infections between 1940 and 1980, sustained by blood transfusion and iatrogenic procedures [5]. In Europe, HCV1b was predominantly found in females and associated with births not later than 1958 [4]. Its prevalence is decreasing due to improved health standards [6]. Interestingly, HCV1b was predominant in Japan, Italy, and Spain with a high prevalence in patients with hepatocellular carcinoma [7]. Since 1997, HCV1b has been the most prevalent subtype in the Calabria Region reflecting national data $[3,8]$.

The major prevalence worldwide and the low susceptibility to Interferon (IFN) or pegylated-IFN alfa with ribavirin (pegIFN- $\alpha / \mathrm{RBV}$ ) therapies made HCV1b the first target for the development of new antiviral drugs [9]. Currently, direct-acting-antiviral (DAA) pan-genotypic therapy can be used to treat infected people without the need for determining the genotype/subtype or performing a resistance test [1]. Pretreatment assessment should consider the presence of cirrhosis and comorbidity in view of post-therapy followup. However, after considering the data of DAA efficacy in a clinical setting, combination therapy still appears to be influenced by resistance-associated substitutions (RASs) carried in target regions in naïve or experienced patients [10].

In this study, we investigated transmission clusters in two cohorts of $\mathrm{HCV} 1 \mathrm{~b}$ positive subjects, enrolled in different time spans, to assess the dynamics of infection in the Calabria Region, southern Italy. In particular, more recent isolates were evaluated for the presence of mutations with a potential impact on treatment response.

\section{Materials and Methods}

\subsection{Study Population}

The study was approved by the Ethical Committee (\#100; 27 April 2017) of the "Mater Domini" University Teaching Hospital of Catanzaro, Italy and it was included in the SINERGIE study [11]. The Ethical Committee approved the criteria that there is no need for informed consent for a non-interventional study. Forty-one serum samples, collected between 1 January 2015 and 31 December 2016, from patients infected by HCV subtype $1 \mathrm{~b}$ were included in the analysis. Enrolled patients, attending the University Hospital of Catanzaro, were randomly selected from a list through a systematic 1:7 sampling procedure. The selected sample is representative of the whole HCV1b cohort, including $41.7 \%$ of males versus $54.0 \%$ of females with an overall median age $68(31-84)$ years [12]. Patients were naïve to all treatments $(25 / 41)$ or treated with IFN (3/41) and pegIFN- $\alpha /$ RBV $(13 / 41)$. Additionally, only viral isolates from HCV1b positive subjects, collected between May and October 2010 during a previous epidemiological study in Calabria, were included in order to compare and investigate phylogenetic relationships with those from Catanzaro. All participants were resident in a small village, Sersale (Catanzaro province) [13]. The patients' clinical data was treated in accordance with the Helsinki Declaration $\left(59^{\text {th }}\right.$ World Medical Association General Assembly, Seoul, Korea, October 2008) and the principles of good clinical practice.

\subsection{Diagnostic Procedures}

HCV RNA viral load was determined using the Cobas AmpliPrep/Cobas TaqMan HCV quantitative test v2.0 (Roche Diagnostics, Milan, Italy). Genotyping was performed by the Versant HCV genotype v2.0 assay (LiPA) (Siemens, Healthcare Diagnostic Inc., Tarrytown, NY, USA). Fibrosis stage was estimated by transient elastometry (FibroScan, Echosens, Paris, France), interpreted as follows: F0-F1 = minimal fibrosis (KPa $\leq 7.1)$, $\mathrm{F} 2=$ moderate fibrosis $(7.1<\mathrm{KPa} \leq 9.5), \mathrm{F} 3=$ severe fibrosis $(9.5<\mathrm{KPa} \leq 14.5), \mathrm{F} 4=$ cirrhosis $(\mathrm{KPa}>14.5)[14]$. 


\subsection{Amplification and Sequencing of HCV NS5B and NS5A Regions}

Viral RNA was extracted from $140 \mu \mathrm{L}$ serum samples using the QIAamp Viral RNA Extraction Kit (Qiagen, Hilden, Germany) in accordance with the manufacturer's instructions. Healthy donor serum samples were used as a negative control. The RNA was reverse transcribed using the High-Capacity cDNA Reverse Transcription Kit protocol (Thermo Fischer Scientific, Waltham, MA, USA) and cDNA amplified by nested PCR using the FastStart High Fidelity PCR system (Roche Diagnostics, Basel, Switzerland). The specific primers used to amplify the NS5B (nt 8256-8632) and NS5A (nt 6086-6722) regions of $\mathrm{HCV}$ genome for the first and second rounds have been previously described $[15,16]$. The products were purified using the High Pure PCR Cleanup Micro Kit (Roche Diagnostics, Basel, Switzerland) and analyzed on 2\% agarose gel stained with GelRed (Biotium Corporete Headquarters, Biotium Inc., Fremont, CA, USA). Both strands were sequenced using the Genome Lab DTCS Quick Start KiT (Beckman Coulter, Inc., Fullerton, CA, USA). Sequencing reactions were run on an automated DNA sequencer (Beckman Coulter, Inc., Fullerton, CA, USA). HCV sequences were aligned by MAFFT under the Galaxy platform (https:/ / usegalaxy.org/, accessed on 27 March 2020) and manually edited by using Bioedit [17-19].

\subsection{Subtyping Tool Analysis}

NS5B and NS5A sequences were analyzed using the Oxford HCV Automated Subtyping Tool v.2.0 (http:/ / dbpartners.stanford.edu/RegaSubtyping/html/subtypinghcvSUB. html, accessed on 20 April 2020) and COMET HCV typing tool (https:/ / comet.lih.lu/index. php?cat=hcv, accessed on 20 April 2020) followed by phylogenetic analysis (see below) to confirm the initial subtyping assignment by LiPA assay [20,21].

\subsection{Datasets Construction}

Two datasets were built. The first dataset contained 78 total sequences: $53 \mathrm{HCV}$ NS5B new sequences from Italy (41 from Catanzaro University Hospital and 12 from Sersale) plus $25 \mathrm{HCV}$ NS5B subtype specific reference sequences downloaded from the HCV Los Alamos sequence database (http://hcv.lanl.gov/content/index, accessed on 11 May 2020). The second dataset comprised 162 total sequences including: $53 \mathrm{HCV}$ NS5B sequences from Italy, previously classified as $1 \mathrm{~b}$ subtype, plus 109 foreign HCV $1 \mathrm{~b}$ NS5B sequences downloaded from the HCV Los Alamos sequence database (http: //hcv.lanl.gov/content/index, accessed on 11 May 2020).

\subsection{Likelihood Mapping}

The phylogenetic signal of each sequence dataset was investigated by means of the likelihood mapping analysis of 10,000 random quartets generated using TreePuzzle [22]. Groups of four randomly chosen sequences (quartets) were evaluated. For each quartet, the three possible unrooted trees were reconstructed using the maximum likelihood approach under the selected substitution model. Posterior probabilities of each tree were then plotted on a triangular surface so that fully resolved trees fell into the corners and the unresolved quartets in the center of the triangle (star-like trees). When using this strategy, if more than $30 \%$ of the dots fall into the center of the triangle, the data is considered unreliable for the purposes of phylogenetic inference.

\subsection{Phylogenetic Analysis}

Sequences of all datasets were aligned using MAFFT under the Galaxy platform and manually edited using Bioedit [17-19]. The subtypes of the newly generated sequences from Calabria were determined and confirmed by phylogenetic analysis of the first dataset. The maximum likelihood phylogenetic trees of the first and second dataset together with the estimation of the best-fit substitution models (TPM2 + F + I + G4 and TVMe + I + G4 for the first and second dataset, respectively) were performed through IQ-TREE with the Model Finder option and visualized with FigTree v. 1.4.4 [23]. Statistical support for 
internal branches of the maximum likelihood (ML) trees were evaluated by bootstrap analysis (1000 replicates) and fast likelihood-based sh-like probability (SH-aLRT).

\subsection{Genetic Variability Analysis}

HCV1b viral population for each patient was screened for genetic variation with a cut-off of $15 \%$ [1]. Forty-one NS5B and twenty-two NS5A sequences at specific nucleotide positions were analyzed. Non-synonymous and resistance-associated substitutions (RAS) were determined using the Geno2pheno (HCV) 0.92 tool (last updated: June 2019) and aligning generated sequences to HCV1b (AJ238799) reference by MAFFT [17,24]. Resistance prediction rules available in the online tool were implemented by literature search [25].

\subsection{Public Availability of the Sequencing Data}

The 41 NS5B and 22 NS5A newly generated sequences were submitted to the GenBank database [26]. All sequences can be retrieved from GenBank under accession numbers: MW357752-MW357814.

\section{Results}

\subsection{Patient Demographic Characteristics and Risk Factors}

The median age of the 53 patients was 70 years (range 31-90), with $58.5 \%$ females. Overall, dental treatment and surgical intervention were the first $(16.9 \%)$ and second $(13.2 \%)$ most frequent risk factors, followed by blood transfusion (3.8\%) and cohabitation (1.9\%). Only one patient reported intravenous drug use as a risk factor. Three patients declared no risk factors. Qualitative characteristics of the two cohorts are reported separately (Table 1).

Table 1. Patient demographic characteristics.

\begin{tabular}{|c|c|c|c|}
\hline \multirow[b]{2}{*}{ Characteristics } & \multicolumn{3}{|c|}{ Absolute Number (\%) } \\
\hline & Overall & $\begin{array}{c}\text { Patients from } \\
\text { University Hospital }\end{array}$ & $\begin{array}{c}\text { Subjects from Sersale } \\
\text { Village }\end{array}$ \\
\hline \multicolumn{4}{|l|}{ Gender } \\
\hline $\mathrm{M}$ & $22(41.5)$ & $21(48.7)$ & $1(8.3)$ \\
\hline $\mathrm{F}$ & $31(58.5)$ & $20(51.3)$ & $11(91.7)$ \\
\hline \multicolumn{4}{|l|}{ Risk factors } \\
\hline Surgical intervention & $7(13.2)$ & $7(17.1)$ & - \\
\hline Blood transfusion & $2(3.8)$ & $2(4.8)$ & - \\
\hline Dental treatment therapy & $9(16.9)$ & $9(21.9)$ & - \\
\hline Cohabitation & $1(1.9)$ & $1(2.4)$ & - \\
\hline Multiple * & $31(58.5)$ & $22(53.6)$ & $9(75)$ \\
\hline Not available & $3(5.7)$ & - & $3(25)$ \\
\hline \multicolumn{4}{|l|}{ Clinical parameters } \\
\hline cirrhotic status & - & $14(34.1)$ & not available \\
\hline HCV RNA median level & $3,792,576 \mathrm{IU} / \mathrm{mL}$ & $2,280,000 \mathrm{IU} / \mathrm{mL}$ & $3,918,625 \mathrm{IU} / \mathrm{mL}$ \\
\hline \multicolumn{4}{|l|}{ Median (range) } \\
\hline Age (years) & $70(31-90)$ & $68(31-85)$ & $71(65-90)$ \\
\hline Total & 53 & 41 & 12 \\
\hline
\end{tabular}

* Multiple risk factors were: surgical intervention + blood transfusion $(n=4)$, surgical intervention + blood transfusion + cohabitation $(n=2)$, blood transfusion + cohabitation $(n=1)$, blood transfusion + dental treatment $(n=2)$, dental treatment + cohabitation $(n=1)$, surgical intervention + cohabitation $(n=2)$, surgical intervention + dental treatment $(n=8)$, surgical intervention + drug user $(n=1)$, surgical intervention + dental treatment + cohabitation $(n=1)$, surgical intervention + glass syringes $(n=7)$, surgical intervention + cohabitation + blood transfusion + glass syringes $(n=1)$, cohabitation + glass syringes $(n=1)$. Characteristics heading and total number of patients were in bold.

\subsection{Likelihood Mapping}

The phylogenetic noise of each dataset was investigated by means of likelihood mapping (Figure S1). The percentage of dots falling in the central area of the triangles was $13.2 \%$ and $7.5 \%$ for the first and second datasets, respectively. As none of the datasets showed more than $30 \%$ of noise, all of them contained sufficient phylogenetic signal. 


\subsection{Phylogenetic Analysis}

All new sequences were classified as subtype $1 \mathrm{~b}$ by both Oxford and COMET subtyping tools, and by phylogenetic analysis. The ML phylogenetic tree of the first dataset showed that all the 53 sequences collected from the Calabria Region were in the same statistically supported clade, closely related to the references subtype $1 \mathrm{~b}$ and were therefore classified as subtype $1 \mathrm{~b}$ (Figure 1 ).

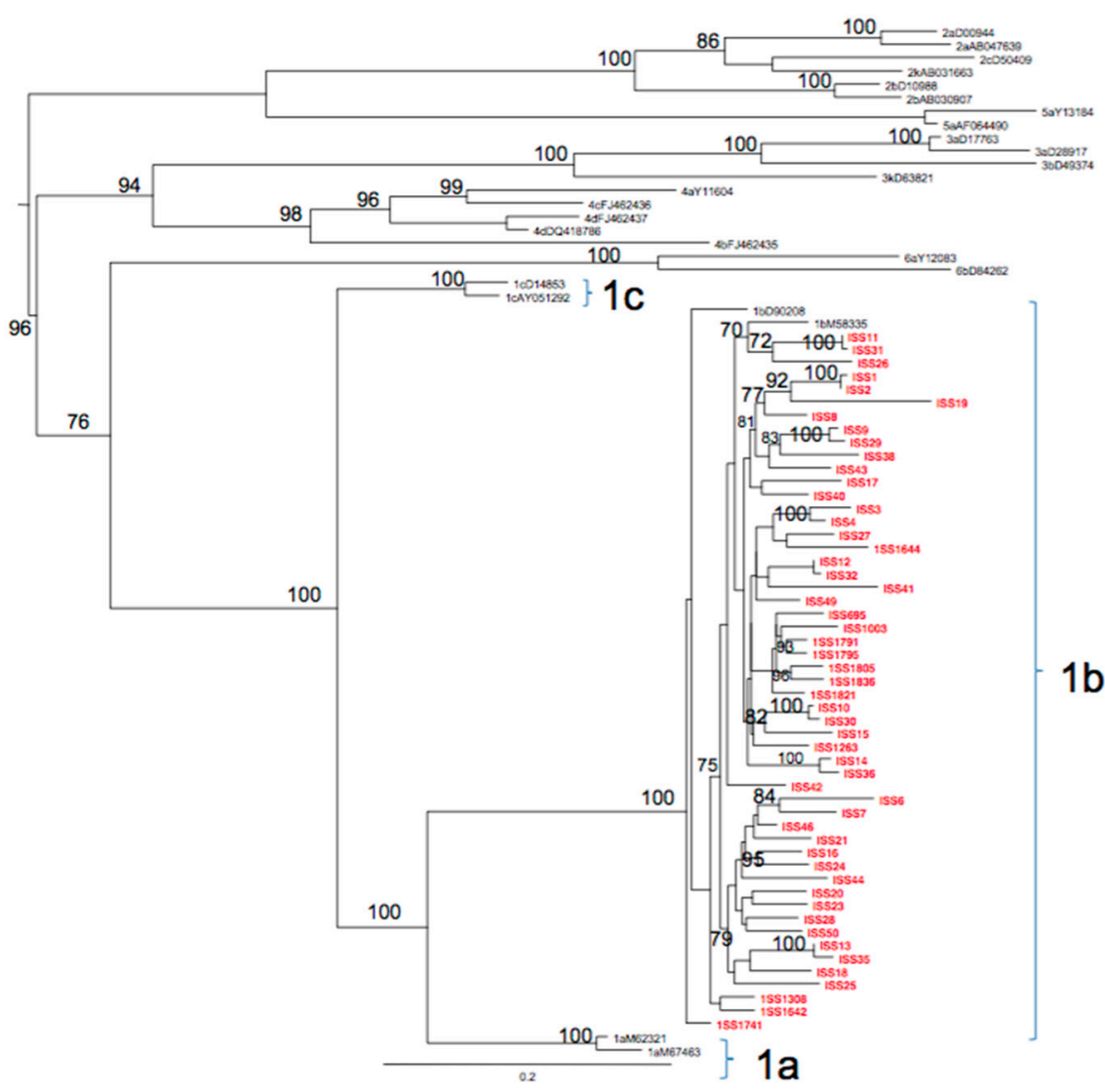

Figure 1. Maximum likelihood phylogenetic tree of the first HCV NS5B dataset. The tree was rooted by using the midpoint rooting method. Branch lengths were estimated with the best fitting nucleotide substitution model according to a hierarchical likelihood ratio test, and were drawn to scale with the bar at the bottom indicating 0.2 nucleotide substitutions per site. The values along a branch represent significant statistical support for the clade subtending that branch (bootstrap support $>75 \%$ ). The Italian (Calabria Region) sequences are highlighted in red. Clades 1b, 1c and 1a are also highlighted with brackets.

The maximum likelihood phylogenetic tree of the second dataset showed the presence of a supported cluster and a main statistically supported clade (Figure 2). 


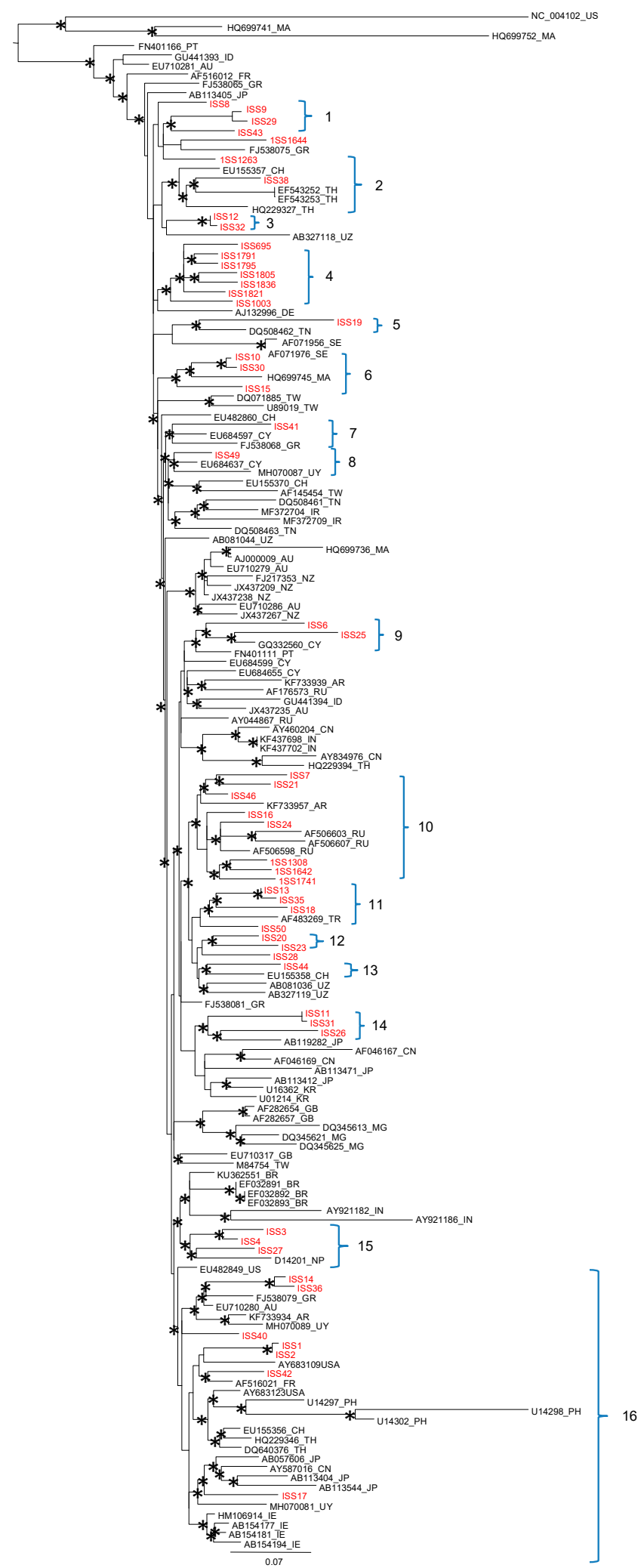

Figure 2. Maximum likelihood phylogenetic tree of the second dataset HCV1b dataset. The tree was rooted by using the midpoint rooting method. Branch lengths were estimated with the best fitting nucleotide substitution model according to a hierarchical likelihood ratio test, and were drawn to scale with the bar at the bottom indicating 0.07 nucleotide substitutions per site. The asterisk $\left({ }^{*}\right)$ along a branch represents significant statistical support for the clade subtending that branch (bootstrap support $>75 \%$ ). The main statistically significant sequences are highlighted with brackets. 
The supported cluster included three foreign (Morocco and US) related sequences. Within the main clade, the HCV1b Italian (Calabria Region) sequences were distributed in 16 statistically supported clusters. Twelve clusters $(12 / 16,75 \%)$, presented foreign HCV $1 \mathrm{~b}$ reference sequences intermixed with sequences from Italy (Clusters: 2, 5, 6, 7, 8, 9, 10, 11, $13,14,15,16)$. Four statistically significant monophyletic clusters, including only sequences from Calabria were also observed (clusters: 1, 3, 4, 12).

Cluster 1 included three sequences from Catanzaro (ISS 9, 29, 43) reporting the following risk factors: blood transfusion/cohabitation, surgery/dental treatment and dental treatment, respectively.

The sequence ISS38 was located in cluster 2 with one reference from Switzerland and three from Thailand. Cluster 3 was composed of two Calabrian sequences (ISS 12 and 32) characterized respectively, by the following risk factors: dental treatment and blood transfusion/dental treatment. Interestingly, cluster 4 included seven sequences (ISS 695; $1791,1795,1805,1836,1821,1003)$, closely related to each other, all from Sersale village. The following risk factors were reported: surgery, cohabitation with HCV positive, sharing glass syringes and blood transfusion (ISS 695), surgery and sharing glass syringes (ISS 1003, 1805, 1821, 1836), no risk factors (ISS 1791, 1795). Cluster 5 included one isolate from Calabria (Catanzaro) reporting blood transfusion as a risk factor and related to a sequence from Tunisia. Cluster 6 was composed of three isolates (ISS10, 30 and 15) from Catanzaro (risk factors: blood transfusion, surgery/dental treatment and surgery) related to a sequence from Morocco. Cluster 7 included one isolate (ISS 41) from Catanzaro characterized by the following risk factor: surgery, related to one reference from Cyprus and one from Greece. Cluster 8 included isolate ISS49 (risk factors: surgery and blood transfusion), one reference from Cyprus and one from Uruguay. Cluster 9 included two isolates from Catanzaro, ISS6 and ISS25 (risk factors: surgery/multiple blood transfusion and surgery/dental treatment, respectively) related to a sequence from Cyprus and another from Portugal. Cluster 10 included eight isolates from Calabria (ISS 7, 21, 46, 16, 24, 1308, 164, 1741), three of which from Sersale, related to one reference from Argentina and three from Russia. Cluster 11 was characterized by three sequences from Catanzaro (ISS 13, 35 and 18) reporting the following risk factors (surgery, surgery/dental treatment, dental treatment, respectively) and related to a reference from Turkey. Cluster 12 included two isolates collected from Catanzaro (ISS 20 and 23) with risk factors: surgery/blood transfusion and surgery/dental treatment, respectively. Cluster 13 was composed of two sequences, the isolate ISS 44 from Catanzaro (reporting surgery/cohabitation as risk factors) related to a reference from Switzerland. Cluster 14 included three isolates from Catanzaro (ISS 11, 31 and 26) reporting the following risk factors (dental treatment, surgery, blood transfusion, and cohabitation) related to a reference from Japan. Cluster 15 included three sequences (ISS 3, 4 and 27) characterized by the following risk factors: surgery/dental treatment; surgery/blood transfusion; surgery/dental treatment/cohabitation and related to a reference from Nepal. Cluster 16 included seven isolates from Catanzaro (ISS 14, 36, 40, 1, 2, 42, 17) intermixed with many sequences sampled from different countries: USA, Greece, Austria, Argentina, Uruguay, France, Philippines, Switzerland, Thailand, Japan, China and Ireland.

\subsection{Substitutions on Target Regions in Patients Nä̈ve to DAA}

The total (100\%) of NS5B amplicons were sequenced. Nine (40\%) NS5A amplicons were not successfully sequenced, while 10 sequences were not of suitable length for RAS screening. Available NS5A and NS5B sequences at the time of genotyping were screened for RASs and nonsynonymous substitutions. We identified the L159F NS5B substitution, conferring resistance to sofosbuvir (SOF), in 15/41 (ISS 6, 7, 13, 16, 18, 20, 21, 23, 24, 25, 28, $35,44,46,50$ ) isolates, among them $8 / 15$ (ISS $6,13,21,24,25,28,35,46$ ) also carried the C316N NS5B related to dasabuvir resistance. In particular, frequency of detected NS5B RASs was $36.6 \%$, while frequency of RASs carried on NS5A region was $22.7 \%$.

The $\mathrm{Y93H}$, associated with resistance to daclatasvir, elbasvir, ledipasvir (LPV), ombitasvir (OMV) and pibrentasvir was detected on NS5A in 3/22 (ISS 16, 24, 30) isolates. The 
L31M substitution associated with resistance to all drugs mentioned above plus velpatasvir was found in 1/22 (ISS 21) isolate. Interestingly, all three isolates carried Y93H plus K108R substitution. The A92T NS5A OMV and LPV associated resistance was detected in 1/22 (ISS 2) isolate. Among patients who reported RASs in the viral population, seven have been previously treated with an IFN regimen with or without RBV and were classified as non-responders (4/7) or relapsers (3/7) with liver stiffness F3 or F4. On the other hand, the $33.3 \%$ of patients without RASs were IFN experienced with or without RBV. The median baseline RNA viral load was 2,280,000 IU/mL.

\section{Discussion}

In order to explore the spread of HCV1b in the Calabria Region, we analyzed NS5B population sequences, obtained from two cohorts of positive individuals, enrolled in different time spans, using phylogenetic analysis. In addition, viral isolates collected between 2015 and 2016 from naïve and IFN/pegIFN- $\alpha$ /RBV treated patients were analyzed in the NS5B and NS5A regions to assess the presence of RASs with the potential to impact on DAA therapy.

Molecular analysis was carried out on 53 sequences of HCV subtype 1b, previously characterized by Inno-Lipa and confirmed by sequencing analysis. As reported in previous studies, subtype $1 b$, together with subtype $2 c$, are the most prevalent genotypes in Italy followed by genotypes 3 and $4[3,6]$. HCV1b diffusion worldwide is related to several risk factors, such as blood transfusions, dental treatment, unsafe reuse of nondisposable syringes $[27,28]$. In previous studies, transmission of two subtypes was already correlated to specific risk factors in the Calabria Region. HCV4d was found related to intravenous drug use and blood transfusion, while HCV2c infection was maintained by unsafe use of glass syringes followed by surgery and unsafe blood transfusion [29,30].

In this work, we investigated possible transmission patterns in a regionally representative sample from a small village (Sersale), where a seminal HCV prevalence study was conducted, and a metropolitan area of the Calabria region [13]. The ML phylogenetic tree shows that the HCV1b Calabria sequences were distributed in 16 statistically supported clusters. Twelve clusters (75\%), contained Italian sequences mixed with foreign HCV1b references while four statistically significant monophyletic clusters included only sequences from Calabria (clusters: 1, 3, 4, 12). In particular, cluster 4 contained only seven closely related Italian sequences collected from Sersale village.

In this study, the majority $(58.5 \%)$ of the enrolled individuals reported multiple risk factors, most of which were surgical intervention and dental treatment $(n=8)$ or surgical intervention and glass syringes $(n=7)$. Individually, we observed that the most frequent risk factors were dental treatment $(16.9 \%)$ and surgical intervention (13.2\%). Interestingly, the risk factors for $\mathrm{HCV}$ acquisition in cluster 4 were medical interventions and multiple use of glass syringes in a family setting as reported in $71 \%$ (no. 5/7) of patients (ISS 695, $1003,1805,1821,1836)$.

Our analysis indicates that in the past, subtype $1 \mathrm{~b}$ was maintained, by sporadic infections, mainly acquired through unsafe use of glass syringes especially in some limited areas of southern Italy, such as Sersale, a small town located 30 miles from Catanzaro. Conversely, in the metropolitan area, other transmission routes, such as dental treatment and surgical intervention had a significant influence on the dissemination of HCV subtype $1 \mathrm{~b}$ throughout the Calabria Region. Interestingly, a community-based survey in the Calabria Region, revealed a high percentage of possible risk factors for $\mathrm{HCV}$ acquisition, such as dental treatment $(69.5 \%)$ and glass syringes injections $(25.8 \%)$ [31].

On the other hand, DAA treatment of hepatitis $C$ could be influenced by baseline RASs naturally occurring in the viral genome [25,32]. It has been reported that $3 \%$ of $\mathrm{HCV}$ positive patients have no virological response, due to the presence of comorbidities and/or RASs in viral isolates, especially in the NS5A viral region [33]. We detected NS5B L159F alone in 15/41 (36.5\%) and in association with C316N in 8/41 (19.5\%) patients, respectively. This last substitution, showing a global frequency of $31.4 \%$ in $\mathrm{HCV} 1 \mathrm{~b}$, is now defined as a 
fitness-associated substitution when combined with the L159F [34]. Therefore, both amino acid variants were associated with a lower response to SOF [35]. Interestingly, NS5B S282T conferring high-level resistance to SOF-containing regimens, was not detected among our isolates, despite being present in $99.1 \%$ of worldwide strains [25]. In three patients, NS5A sequences carried the $\mathrm{Y} 93 \mathrm{H}$ substitution, currently the major clinically relevant RAS contributing to failure of many approved IFN-free regimens [36]. Additionally, all three isolates showed the $\mathrm{Y} 93 \mathrm{H}+\mathrm{K} 108 \mathrm{R}$ profile, which is associated with a minor affinity to OMV drug with respect to the $\mathrm{Y} 93 \mathrm{H}+\mathrm{R} 108 \mathrm{~K}$ combination as previously reported [37]. However, the $97 \%$ of treated patients with DAAs achieved sustained virological response (SVR). According to our experience about a single-center cohort in Southern Italy, the SVR rate was $97 \%$ for the older age group, $96 \%$ for people under 65 years old, finally $94 \%$ and $100 \%$ for cirrhotic and non-cirrhotic patients, respectively [38].

\section{Conclusions}

Despite the sample size being a limitation of the study, this suggests that the spread of HCV1b was maintained in the Calabria Region by sporadic infections, mainly acquired through the unsafe use of glass syringes, dental treatment and surgical intervention. Even if our analysis was performed on samples collected in 2015-2016, the frequency of natural RASs carried on subtype-specific viral strains, as well as comorbidities of treated patients, should be taken into account for the effectiveness of IFN-free regimens to eradicate $\mathrm{HCV}$ infection.

Supplementary Materials: The following are available online at https:/ / www.mdpi.com/article/10 .3390/jcm10081655/s1, Figure S1: Likelihood mapping of HCV NS5B first (a) and second (b) dataset.

Author Contributions: A.R.C. and R.B.: conceptualization. A.C., S.T., M.E., A.L.P., G.P., G.S.B., A.Q., G.M.: methodology. A.C., S.T., M.E., A.L.P., G.P., N.M.: A.C., S.T., M.E., G.P.: formal analysis. investigation. E.M.T., C.C., M.M., F.S., C.T.: data curation. A.C., S.T., A.L.P., N.M.: writing—original draft preparation. A.R.C., M.C.L., C.T., N.M.: writing-review and editing. A.R.C.: supervision. All authors have read and agreed to the published version of the manuscript.

Funding: This research received no external funding.

Institutional Review Board Statement: The study was conducted according to the guidelines of the Declaration of Helsinki, and approved by the Ethical Committee (\#100; 27 April 2017) of the "Mater Domini" University Teaching Hospital of Catanzaro, Italy.

Informed Consent Statement: Patient consent was waived due to non-interventional study. The Ethical Committee approved the criteria that there is no need for informed consent for a noninterventional study.

Data Availability Statement: The 41 NS5B and 22 NS5A newly generated sequences used for molecular analysis have been uploaded to GenBank under the following accession numbers: MW357752MW357814. Materials supporting the findings of this study are available within the article.

Acknowledgments: The authors would like to thank Neill J. Adams (Department of Health Sciences, Institute of Microbiology, "Magna Grecia" University of Catanzaro, Italy) who checked the English throughout the manuscript. Maria Mazzitelli was supported as PhD student by European Commission (FESR FSE 2014-2020) and by Calabria Region (Italy). European Commission and Calabria Region cannot be held responsible for any use, which may be made of information contained therein.

Conflicts of Interest: The authors declare no conflict of interest.

\section{References}

1. European Association for the Study of the Liver. EASL Recommendations on Treatment of Hepatitis C 2020. J. Hepatol. 2020, 73, 1170-1218.

2. Borgia, S.M.; Hedskog, C.; Parhy, B.; Hyland, R.H.; Stamm, L.M.; Brainard, D.M.; Subramanian, M.G.; McHutchison, J.G.; Mo, H.; Svarovskaia, E.; et al. Identification of a Novel Hepatitis C Virus Genotype From Punjab, India: Expanding Classification of Hepatitis C Virus Into 8 Genotypes. J. Infect. Dis. 2018, 218, 1722-1729. [CrossRef] 
3. Marascio, N.; Liberto, M.C.; Barreca, G.S.; Zicca, E.; Quirino, A.; Lamberti, A.G.; Bianco, G.; Matera, G.; Surace, L.; Berardelli, G.; et al. Update on epidemiology of HCV in Italy: Focus on the Calabria Region. BMC Infect. Dis. 2014, 14, S2. [CrossRef] [PubMed]

4. Kartashev, V.; Döring, M.; Nieto, L.; Coletta, E.; Kaiser, R.; Sierra, S.; Guerrero, A.; Stoiber, H.; Paar, C.; Vandamme, A.; et al. New findings in HCV genotype distribution in selected West European, Russian and Israeli regions. J. Clin. Virol. 2016, 81, 82-89. [CrossRef] [PubMed]

5. Magiorkinis, G.; Magiorkinis, E.; Paraskevis, D.; Ho, S.Y.W.; Shapiro, B.; Pybus, O.G.; Allain, J.-P.; Hatzakis, A. The Global Spread of Hepatitis C Virus 1a and 1b: A Phylodynamic and Phylogeographic Analysis. PLoS Med. 2009, 6, e1000198. [CrossRef]

6. Ansaldi, F.; Bruzzone, B.; Salmaso, S.; Rota, M.C.; Durando, P.; Gasparini, R.; Icardi, G. Different seroprevalence and molecular epidemiology patterns of hepatitis C virus infection in Italy. J. Med. Virol. 2005, 76, 327-332. [CrossRef] [PubMed]

7. Mitra, A.K. Hepatitis C-related hepatocellular carcinoma: Prevalence around the world, factors interacting, and role of genotypes. Epidemiol. Rev. 1999, 21, 180-187. [CrossRef] [PubMed]

8. Liberto, M.C.; Marascio, N.; Zicca, E.; Matera, G. Epidemiological features and specificities of HCV infection: A hospital-based cohort study in a university medical center of Calabria region. BMC Infect. Dis. 2012, 12, S4. [CrossRef]

9. Cuypers, L.; Snoeck, J.; Vrancken, B.; Kerremans, L.; Vuagniaux, G.; Verbeeck, J.; Nevens, F.; Camacho, R.J.; Vandamme, A.M.; Van Dooren, S. A near-full length genotypic assay for HCV1b. J. Virol. Methods 2014, 209, 126-135. [CrossRef]

10. Marascio, N.; Quirino, A.; Barreca, G.S.; Galati, L.; Costa, C.; Pisani, V.; Mazzitelli, M.; Matera, G.; Liberto, M.C.; Focà, A.; et al. Discussion on critical points for a tailored therapy to cure hepatitis C virus infection. Clin. Mol. Hepatol. 2019, 25, 30-36. [CrossRef]

11. Torti, C.; Zazzi, M.; Abenavoli, L.; Trapasso, F.; Cesario, F.; Corigliano, D.; Cosco, L.; Costa, C.; Curia, R.L.; De Rosa, M.; et al. SINERGIE Study Group. Future research and collaboration: The "SINERGIE" project on HCV (South Italian Network for Rational Guidelines and International Epidemiology). BMC Infect. Dis. 2012, 12, S9. [CrossRef] [PubMed]

12. Marascio, N.; Mazzitelli, M.; Scarlata, G.G.M.; Giancotti, A.; Barreca, G.S.; Lamberti, A.G.; Divenuto, F.; Costa, C.; Trecarichi, E.M.; Matera, G.; et al. HCV Antibody Prevalence and Genotype Evolution in a Teaching Hospital, Calabria Region, Southern Italy Over A Decade (2008-2018). Open Microbiol. 2020, 14, 84-90. [CrossRef]

13. Guadagnino, V.; Stroffolini, T.; Caroleo, B.; Menniti Ippolito, F.; Rapicetta, M.; Ciccaglione, A.R.; Chionne, P.; Madonna, E.; Costantino, A.; De Sarro, G.; et al. Hepatitis C virus infection in an endemic area of Southern Italy 14 years later: Evidence for a vanishing infection. Dig. Liver Dis. 2013, 45, 403-407. [CrossRef] [PubMed]

14. Castera, L.; Vergniol, J.; Foucher, J.; Le Bail, B.; Chanteloup, E.; Haaser, M.; Darriet, M.; Couzigou, P.; de Ledinghen, V. Prospective comparison of transient elastography, Fibrotest, APRI, and liver biopsy for the assessment of fibrosis in chronic hepatitis C. Gastroenterology 2005, 128, 343-350. [CrossRef]

15. Pybus, O.G.; Barnes, E.; Taggart, R.; Lemey, P.; Markov, P.V.; Rasachak, B.; Syhavong, B.; Phetsouvanah, R.; Sheridan, I.; Humphreys, I.S.; et al. Genetic history of hepatitis C virus in East Asia. J. Virol. 2009, 83, 1071-1082. [CrossRef]

16. Lindström, I.; Kjellin, M.; Palanisamy, N.; Bondeson, K.; Wesslén, L.; Lannergard, A.; Lennerstrand, J. Prevalence of polymorphisms with significant resistance to NS5A inhibitors in treatment-naive patients with hepatitis C virus genotypes 1a and 3a in Sweden. Infect. Dis. 2015, 47, 555-562. [CrossRef]

17. Katoh, K.; Standley, D.M. MAFFT multiple sequence alignment software version 7: Improvements in performance and usability. Mol. Biol. Evol. 2013, 30, 772-780. [CrossRef]

18. Afgan, E.; Baker, D.; Batut, B.; van den Beek, M.; Bouvier, D.; Cech, M.; Chilton, J.; Clements, D.; Coraor, N.; Grüning, B.A.; et al. The Galaxy platform for accessible, reproducible and collaborative biomedical analyses: 2018 update. Nucleic Acids Res. 2018, 46, W537-W544. Available online: https:/ / usegalaxy.org/ (accessed on 27 March 2020). [CrossRef]

19. Hall, T.A. BioEdit: A User-Friendly Biological Sequence Alignment Editor and Analysis Program for Windows 95/98/NT. Nucleic Acids Symp. Ser. 1999, 41, 95-98.

20. de Oliveira, T.; Deforche, K.; Cassol, S.; Salminen, M.; Paraskevis, D.; Seebregts, C.; Snoeck, J.; van Rensburg, E.J.; Wensing, A.M.; van de Vijver, D.A.; et al. An automated genotyping system for analysis of HIV-1 and other microbial sequences. Bioinformatics 2005, 21, 3797-3800. Available online: http:/ / dbpartners.stanford.edu/RegaSubtyping/html/subtypinghcvSUB.html (accessed on 20 April 2020). [CrossRef]

21. Struck, D.; Lawyer, G.; Ternes, A.M.; Schmit, J.C.; Perez Bercoff, D. COMET: Adaptive context-based modeling for ultrafast HIV-1 subtype identification. Nucleic Acids Res. 2014, 42, e144. Available online: https:/ / comet.lih.lu/index.php?cat=hcv (accessed on 20 April 2020). [CrossRef]

22. Schmidt, H.A.; Strimmer, K.; Vingron, M.; von Haeseler, A. TREE-PUZZLE: Maximum likelihood phylogenetic analysis using quartets and parallel computing. Bioinformatics 2002, 18, 502-504. [CrossRef]

23. Nguyen, L.T.; Schmidt, H.A.; von Haeseler, A.; Minh, B.Q. IQ-TREE: A fast and effective stochastic algorithm for estimating maximum likelihood phylogenies. Mol. Biol. Evol. 2015, 32, 268-274. [CrossRef]

24. Kalaghatgi, P.; Sikorski, A.M.; Knops, E.; Rupp, D.; Sierra, S.; Heger, E.; Neumann-Fraune, M.; Beggel, B.; Walker, A.; Timm, J.; et al. Geno2pheno[HCV]—A web-based interpretation system to support hepatitis C treatment decisions in the era of direct-acting antiviral agents. PLoS ONE 2016, 11, 1416. [CrossRef] [PubMed]

25. Di Maio, V.C.; Cento, V.; Lenci, I.; Aragri, M.; Rossi, P.; Barbaliscia, S.; Melis, M.; Verucchi, G.; Magni, C.F.; Teti, E.; et al. HCV Italian Resistance Network Study Group. Multiclass HCV resistance to direct-acting antiviral failure in real-life patients advocates for tailored second-line therapies. Liver Int. 2017, 37, 514-528. [CrossRef] 
26. Benson, D.A.; Clark, K.; Karsch-Mizrachi, I.; Lipman, D.J.; Ostell, J.; Sayers, E.W. GenBank. Nucleic Acids Res. $2014,42,32-37$. [CrossRef] [PubMed]

27. Alter, M.J. HCV routes of transmission: What goes around comes around. Semin. Liver Dis. 2011, 31, 340-346. [CrossRef] [PubMed]

28. Olmstead, A.D.; Joy, J.B.; Montoya, V.; Luo, I.; Poon, A.F.; Jacka, B.; Lamoury, F.; Applegate, T.; Montaner, J.; Khudyakov, Y.; et al. A molecular phylogenetics-based approach for identifying recent hepatitis C virus transmission events. Infect. Genet. Evol. 2015, 33, 101-109. [CrossRef]

29. Ciccozzi, M.; Equestre, M.; Costantino, A.; Marascio, N.; Quirino, A.; Lo Presti, A.; Cella, E.; Bruni, R.; Liberto, M.C.; Focà, A.; et al. Hepatitis C virus genotype $4 \mathrm{~d}$ in Southern Italy: Reconstruction of its origin and spread by a phylodynamic analysis. J. Med. Virol. 2012, 84, 1613-1619. [CrossRef]

30. Marascio, N.; Ciccozzi, M.; Equestre, M.; Lo Presti, A.; Costantino, A.; Cella, E.; Bruni, R.; Liberto, M.C.; Pisani, G.; Zicca, E.; et al. Back to the origin of HCV 2c subtype and spreading to the Calabria region (Southern Italy) over the last two centuries: A phylogenetic study. Infect. Genet. Evol. 2014, 26, 352-358. [CrossRef]

31. Serraino, R.; Mazzitelli, M.; Greco, G.; Serapide, F.; Scaglione, V.; Marascio, N.; Trecarichi, E.M.; Torti, C. Risk factors for hepatitis B and $\mathrm{C}$ among healthy population: A community-based survey from four districts of Southern Italy. Infez. Med. 2020, 28, 223-226.

32. Marascio, N.; Pavia, G.; Strazzulla, A.; Dierckx, T.; Cuypers, L.; Vrancken, B.; Barreca, G.S.; Mirante, T.; Malanga, D.; Oliveira, D.M.; et al. The SINERGIE-UMG Study Group. Detection of Natural Resistance-Associated Substitutions by Ion Semiconductor Technology in HCV1b Positive, Direct-Acting Antiviral Agents-Naïve Patients. Int. J. Mol. Sci. 2016, 17, 1416. [CrossRef]

33. Calvaruso, V.; Petta, S.; Craxì, A. Is global elimination of HCV realistic? Liver Int. 2018, 38, 40-46. [CrossRef] [PubMed]

34. Cuypers, L.; Li, G.; Libin, P.; Piampongsant, S.; Vandamme, A.M.; Theys, K. Genetic Diversity and Selective Pressure in Hepatitis C Virus Genotypes 1-6: Significance for Direct-Acting Antiviral Treatment and Drug Resistance. Viruses 2015, 7, 5018-5039. [CrossRef] [PubMed]

35. Gaspareto, K.V.; Ribeiro, R.M.; de Mello Malta, F.; Gomes-Gouvêa, M.S.; Muto, N.H.; Romano, C.M.; Mendes-Correa, M.C.; Carrilho, F.J.; Sabino, E.C.; Rebello Pinho, J.R. Resistance-associated variants in HCV subtypes 1a and 1b detected by Ion Torrent sequencing platform. Antivir. Ther. 2016, 21, 653-660. [CrossRef] [PubMed]

36. Zeuzem, S.; Mizokami, M.; Pianko, S.; Mangia, A.; Han, K.H.; Martin, R.; Svarovskaia, E.; Dvory-Sobol, H.; Doehle, B.; Hedskog, C.; et al. NS5A resistance-associated substitutions in patients with genotype 1 hepatitis $C$ virus: Prevalence and effect on treatment outcome. J. Hepatol. 2017, 66, 910-918. [CrossRef] [PubMed]

37. Marascio, N.; Pavia, G.; Romeo, I.; Talarico, C.; Di Salvo, S.; Reale, M.; Marano, V.; Barreca, G.S.; Fabiani, F.; Perrotti, N.; et al. Real-life 3D therapy failure: Analysis of NS5A 93H RAS plus $108 \mathrm{~K}$ polymorphism in complex with ombitasvir by molecular modeling. J. Med. Virol. 2018, 90, 1257-1263. [CrossRef]

38. Scaglione, V.; Mazzitelli, M.; Costa, C.; Pisani, V.; Greco, G.; Serapide, F.; Lionello, R.; La Gamba, V.; Marascio, N.; Trecarichi, E.M.; et al. Virological and Clinical Outcome of DAA Containing Regimens in a Cohort of Patients in Calabria Region (Southern Italy). Medicina 2020, 56, 101. [CrossRef] 